\title{
MODELO DE DIFUSÃO DE BASS: UMA APLICAÇÃO PARA A INDÚSTRIA DE MOTOCICLETAS NO BRASIL
}

\section{BASS DIFFUSION MODEL: AN APPLICATION TO THE INDUSTRY OF MOTORCYCLES IN BRAZIL}

\author{
Júlio César Bastos de Figueiredo \\ Escola Superior de Propaganda e Marketing - ESPM - São Paulo - Brasil \\ jfigueiredo@espm.br
}

\begin{abstract}
Resumo
Este trabalho tem como objetivo discutir e aplicar o modelo de difusão de Frank Bass para o estudo e previsão dos ciclos de difusão de marcas e modelos de motocicletas comercializadas no Brasil. O trabalho analisa, comparativamente, o processo de difusão de diferentes marcas $e$ modelos de motocicletas nas categorias de 50 e 600 cilindradas. Isso será feito tentando-se responder, por meio da análise dos parâmetros dos modelos construídos, quais mecanismos de difusão: inovação ou contaminação social; são mais impactantes na comercialização dos diversos modelos e marcas. Será utilizado o método de mínimos quadrados não lineares (MQNL) no ajuste dos dados históricos de vendas de diversos modelos de motocicletas ao modelo de Frank Bass. Como resultado, mostra-se que modelo de Frank Bass pode ser utilizado para reproduzir curvas de vendas de motocicletas, e que os parâmetros fornecidos pelo ajuste podem ser utilizados para melhor compreender a dinâmica deste processo.
\end{abstract}

Palavras-chave: difusão de inovações; modelo de Bass; motocicletas.

\section{Introdução}

Para que um novo produto, modelo ou marca se torne economicamente viável em um novo mercado é necessário possuir profundo conhecimento não apenas dos processos de desenvolvimento técnico, mas também dos estágios típicos de penetração e difusão do produto nos mercados consumidores (FIGUEIREDO, 2009a; PERES; MULLER et al., 2010).

O uso de modelos matemáticos de difusão de novos produtos permite aos gestores a definição de formas mais seguras e objetivas para lidar com suas expectativas relativas a vendas futuras. Além disso, análises estruturadas do processo de difusão, como a que será apresentada neste trabalho, podem evitar, ou pelo menos ajudar a minimizar, problemas na formulação de estratégias de comercialização em mercados com ciclos rápidos de desenvolvimento e lançamento (FIGUEIREDO, 2009a; b), como acontece no setor de motocicletas no Brasil. Este trabalho busca discutir a importância para as empresas deste setor, principalmente aquelas envolvidas em 
processos de pesquisa e desenvolvimento, do estudo dos estágios típicos de penetração e difusão de novos produtos.

Rogers (1995) define a difusão como sendo o processo pelo qual uma inovação espalha-se entre os membros de um sistema social. Para Rogers, toda a comunicação de uma inovação entre os membros de um particular sistema social caracteriza um processo de difusão. Para Stoneman (2002) o termo difusão pode ser definido objetivamente como o crescimento do uso ou consumo de um bem ou serviço dentro de um determinado grupo social. Neste contexto, o objetivo dos modelos de difusão é o de descrever o ciclo de vida de um novo produto. Os modelos de difusão focam no desenvolvimento de uma curva do ciclo de vida e atingem o propósito de previsão de vendas de um novo produto. Modelos de difusão, por definição, visam representar o crescimento de uma categoria de produto (MAHAJAN; VIJAY; MULLER; EITAN et al., 1990).

O lançamento de novos produtos afeta a vida de todos os indivíduos e comunidades. Todavia, o consumo destes produtos não cresce instantaneamente a partir de seu lançamento. Ao contrário, as vendas dos produtos tipicamente evoluem nos mercados de acordo com uma curva que consiste basicamente de quatro estágios (Figura 1): (a) uma etapa introdutória de pequeno crescimento; (b) um estágio com crescimento muito rápido; (c) um estágio de maturidade, que é tipicamente marcado por pouco ou mesmo nenhum crescimento, e (d) uma fase final de declínio, onde as vendas caem devido à obsolescência ou a substituição (MAHAJAN; VIJAY; MULLER; EITAN et al., 1990).

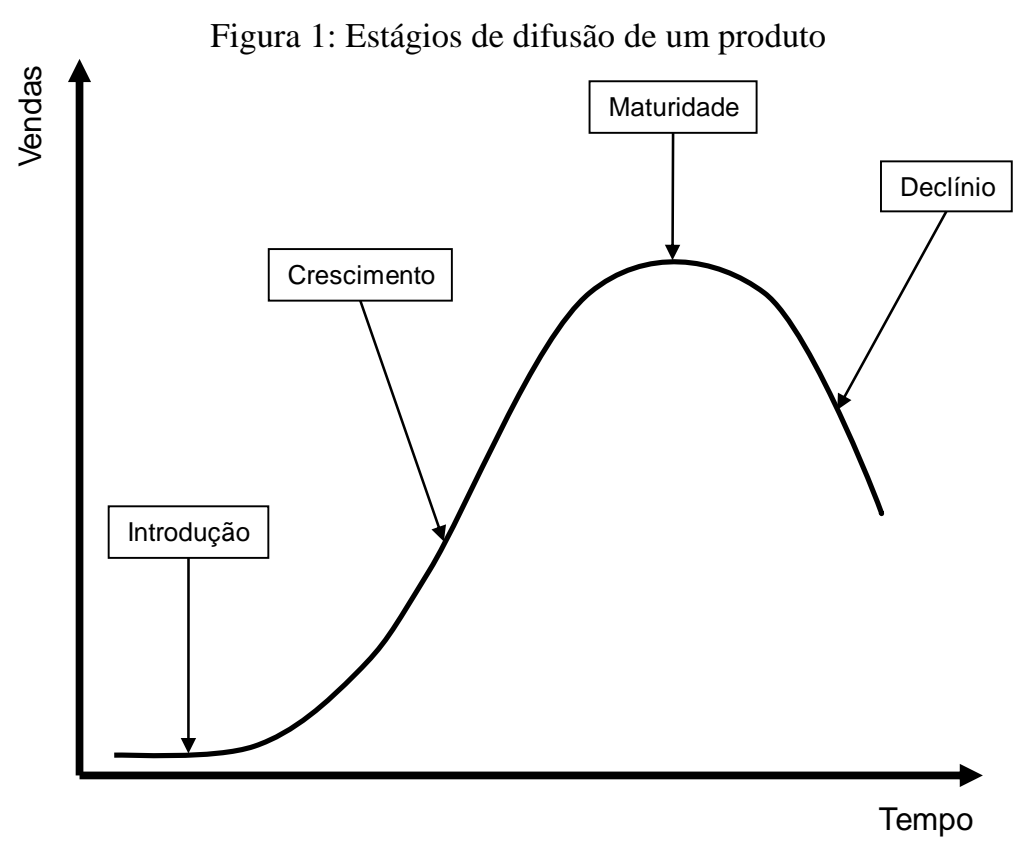

Fonte: Elaborado pelo autor

Os primeiros trabalhos sobre a difusão de produtos surgiram na década de 60. Os primeiros modelos foram propostos por Fourt e Woodlock (1960). Estes autores assumiram que o processo de 
difusão é principalmente influenciado pela comunicação de massa. Outro autor, Mansfield (1961), assumiu que o processo é influenciado principalmente pela comunicação interpessoal (boca a boca). Estes trabalhos serviram como base para que Frank Bass (1969) desenvolve-se um modelo de difusão abrangendo os princípios propostos por Fourt e Woodlock (1960) e Mansfield (1961). O modelo de Frank Bass assume que os consumidores potenciais de uma inovação são influenciados por dois processos: a comunicação de massa e a comunicação interpessoal.

Desde sua publicação em 1969, o modelo de Bass tem sido o modelo de difusão mais utilizado no estudo da difusão de novos produtos, tanto em problemas locais quanto em estudos que envolvem a difusão de novos produtos através de diversos países. Ele é capaz de gerar uma boa representação de quase todos os processos de difusão de novos produtos, mesmo em uma realidade onde é grande a quantidade de fatores que influenciam o comportamento do mercado.

Este trabalho se justifica por dois aspectos: em primeiro lugar demonstra que o modelo de difusão de Frank Bass pode ser utilizado como instrumento de projeção das curvas de vendas de modelos de motocicletas no Brasil. Isto é importante porque o modelo de Frank Bass permite saber, por meio da análise dos parâmetros ajustados, quais mecanismos de difusão: inovação ou contaminação social; são mais impactantes na penetração dos modelos e das marcas. Esta informação é particularmente relevante para a construção de estratégias eficazes de marketing e comunicação (CHANDRASEKARAN; TELLIS, 2006). Em segundo lugar, a pesquisa também é justificada por que considera questões que envolvem um mercado importante no Brasil. Como é um setor onde os processos de evolução tecnológica são contínuos, levando a grandes investimentos técnicos e de infraestrutura, as montadoras necessitam frequentemente efetuar estudos sobre a evolução da demanda para formular estratégias de criação de oferta. Dessa forma, os modelos apresentado neste trabalho poderão auxiliar no dimensionamento de investimentos futuros a serem realizados por essas empresas.

\section{O modelo de difusão de Frank Bass}

O modelo de Frank Bass (1969) é um dos modelos matemáticos mais utilizados no estudo da difusão de novos produtos. Sua construção e seu comportamento racional são consistentes com diversos estudos no campo da ciência social, e sua eficácia tem sido extensivamente comprovada ao longo dos anos (WRIGHT; CHARIETT, 1995).

O modelo de Bass nasce de uma hipótese comportamental na qual se assume que, durante o processo de difusão do uso de um novo produto, dois tipos de consumidores irão determinar a forma como a demanda irá crescer. $\mathrm{O}$ primeiro tipo corresponde àqueles indivíduos que decidem adotar o produto de forma independentemente, ou seja, não recebem influência direta de outros consumidores, todavia, podem ser motivados por outros meios, por exemplo, por comunicação de 
massa. Esses indivíduos são denominados "inovadores". O segundo tipo corresponde aos consumidores potenciais que são influenciados pela pressão social do meio e que são susceptíveis à influência de outros consumidores que já adquiriram o produto. Estes consumidores são denominados "imitadores".

Esta hipótese comportamental pode ser transcrita diretamente em um modelo matemático. Considere $S(t)$ como sendo o total acumulado no instante $t$ de consumidores que já adquiriram um determinado produto em um mercado de $m$ consumidores potenciais. No modelo de Bass assume-se de forma simplificada que a "pressão de adoção" - $P(t)$, que corresponde à probabilidade de compra no instante $t$, por um consumidor aleatório, é uma função linear da proporção de usuários que já adotaram o produto, ou seja:

$$
P(t)=p+q \cdot \frac{S(t)}{m} .
$$

A Equação (1) corresponde à premissa fundamental do modelo de Bass. Se verdadeira, então o parâmetro $p$ corresponderá à tendência autônoma (intrínseca) do indivíduo em adotar o novo produto, geralmente estimulada pelo processo de comunicação (HORSKY; SIMON, 1983; KALISH, 1985; KALISH; LILIEN, 1986). Este parâmetro é comumente chamado na literatura de "coeficiente de inovação". Já o parâmetro q é um parâmetro que está ligado a uma tendência de adoção motivada pelo que é chamado de "contágio social”.

Observe que o segundo termo da Equação (1) crescerá na medida em que aumentar a proporção de indivíduos no mercado que adotam o produto, $(\mathrm{S}(t) / m)$, e este crescimento será proporcional ao parâmetro $q$. O parâmetro $q$ corresponde então a uma força de contágio, e é por isso que é chamado na literatura de "coeficiente de imitação".

Uma vez que $S(t)$ é o total acumulado no instante $t$ de consumidores que já adquiriram um determinado produto, $d S(t) / d t$ será a taxa de acréscimo de novos consumidores a cada instante de tempo. Logo, a proporção de indivíduos no instante $t$ que adotam o produto, em relação àqueles que ainda não adotaram no mercado, será dada por:

$$
h(t)=\left(\frac{1}{m-S(t)}\right) \cdot \frac{d}{d t} S(t)
$$

Onde $m$ corresponde ao número total de consumidores potenciais no mercado.

Combinando a Equação (2) com a Equação (1), e definido $U(t)=(m-S(t))$ como sendo o número de consumidores potenciais que ainda restam adquirir o produto, obtém-se o modelo de 
Bass na sua forma diferencial:

$$
\frac{d}{d t} S(t)=p \cdot U(t)+q \cdot U(t) \cdot \frac{S(t)}{m}
$$

Assumindo-se que $F(t)=N(t) / m$ seja a fração de adotantes potenciais no instante $t$, o modelo de Bass pode ser expresso então como:

$$
\frac{d}{d t} F(t)=(p+q F(t))(1-F(t)) .
$$

A curva $\mathrm{S}(\mathrm{t})$ obtida pela solução da Equação (3) apresenta um padrão de crescimento logístico com saturação em $\mathrm{S}(\mathrm{t})=\mathrm{m}$ (Figura 2). Em razão disto, $m$ também é chamado de "capacidade de carga" do mercado.

Uma série métodos estimativos têm sido sugeridos para estimar os parâmetros p, q e m do modelo de Bass. Mahajan et al. (1990) compararam quatro métodos de estimativa: Mínimos Quadrados Ordinários (MQO), Estimativa por Máxima Verossimilhança (EMV), Mínimos Quadrados Não Lineares (MQNL) e Estimação Algébrica (EA). Eles concluíram que o método MQNL é o que levava às melhores estimativas e predições, além de ser um método ser de fácil implementação.

Figura 2: Representação genérica de uma solução da Equação (3)

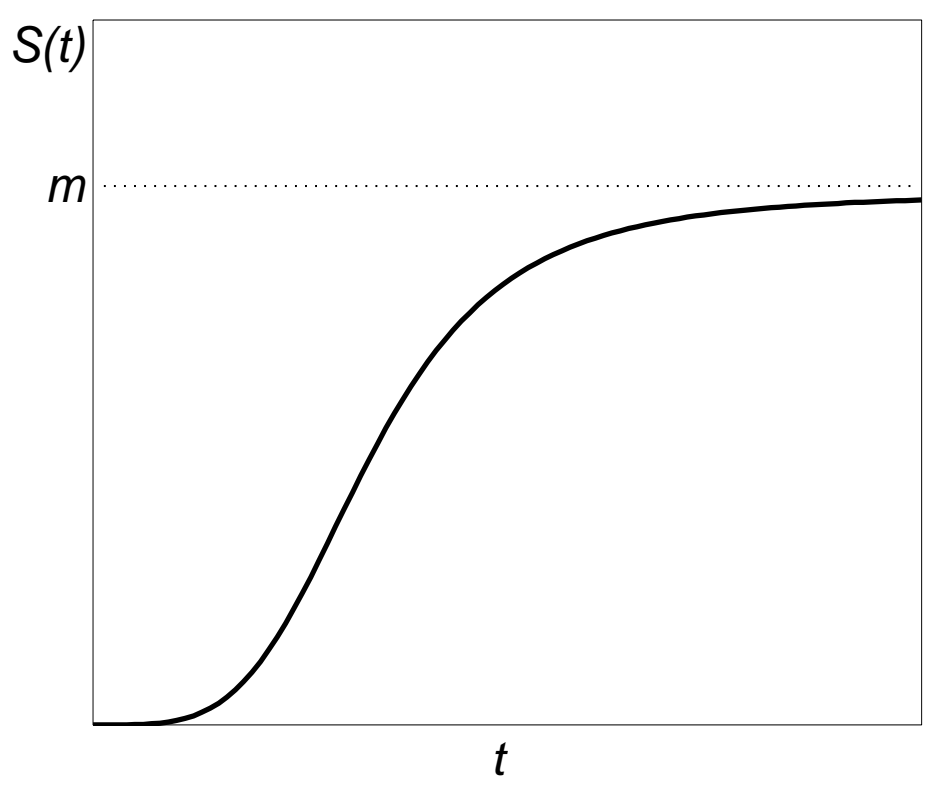

Fonte: Elaborado pelo autor 
Em relação ao número de períodos necessários para que o modelo possa ser ajustado, Srinivasan e Mason (1986) mostraram ainda que as estimativas do modelo de Bass ficam comprometidas se menos de 4 períodos anuais são disponíveis para o ajuste. Heeler e Hustad (1980) recomendam pelo menos 10 períodos anuais que incluam o pico das vendas. Neste trabalho é feito o ajuste do modelo de Bass apenas para séries temporais de vendas com mais de 5 anos que incluam o pico de vendas. É também utilizado o método de mínimos quadrados não lineares proposto por Srinivasan e Mason (1986). Este método têm algumas vantagens sobre outro por superar um problema relativo às estimativas de intervalos discretos, comum nos métodos de mínimos quadrados ordinários (MQO) (SATOH, 2001).

Srinivasan e Mason (1986) sugerem que os estimadores $\hat{p}, \hat{q}$ e $\hat{m}$ podem então ser obtidos utilizando a seguinte expressão para o número de adotantes $N(i)$ :

$$
N(i)=m\left(\frac{1-e^{-(p+q) t_{i}}}{1+(q / p) e^{-(p+q) t_{i}}}-\frac{1-e^{-(p+q) t_{i-1}}}{1+(q / p) e^{-(p+q) t_{i-1}}}\right)+\mu_{i}
$$

Onde $\mu_{i}$ é um termo aditivo de erro.

A Equação (5) é ajustada para cada conjunto de dados utilizando um algoritmo de MNQL. Cada ajuste fornecerá o conjunto de parâmetros $p, q$ e $m$ que melhor descreve a curva de Bass subjacente à difusão do modelo de motocicleta estudado.

\section{O mercado de motos no Brasil}

A indústria automotiva data da década de 50, e hoje se constitui em uma das maiores do mundo. Já o mercado de motos no Brasil tem início na década de 60 (VASCONCELLOS, 2008), sendo que as motocicletas não foram economicamente relevantes até os anos 1990, quando começaram os processos de liberalização econômica e privatização. Em 1990, o Brasil tinha 20,6 milhões de veículos e apenas 1,5 milhão de motocicletas. Algumas motocicletas eram fabricadas no país e outras eram importadas dos EUA ou do Japão, sendo que elas eram usadas principalmente pelas pessoas de renda mais alta, por motivo de lazer.

Com o Plano Real, em 1994, houve um grande processo de abertura econômica no Brasil, associado a intensas transformações em escala global. O Brasil passou a conviver com forças poderosas de desregulamentação e privatização que afetaram profundamente o país e a forma de distribuição dos seus recursos. Segundo a Associação Brasileira dos Fabricantes de Motocicletas, Ciclomotores, Motonetas, Bicicletas e Similares - ABRACICLO, a frota de 1,5 milhão de motocicletas em 1991 rapidamente aumentou para 5 milhões em 2002, chegando a mais de 13 
milhões em 2010 (ABRACICLO, 2010).

Os dados da ABRACICLO mostram também que as motos venderam em 2010 aproximadamente 1.800.000 unidades (ABRACICLO, 2010) contra as 2.600.000 unidades de automóveis computadas pela Associação Nacional dos Fabricantes de Veículos Automotores ANFAVEA - no mesmo período (ANFAVEA, 2010). Ou seja, as vendas anuais de motos cada vez mais se aproximam em volume das de automóveis.

O mercado de motocicletas é hoje responsável no Brasil por mais de 150 mil empregos diretos e indiretos. Com 11 fabricantes, instalados na Zona Franca de Manaus, vem crescendo a taxas superiores a 10\% ao ano nos últimos 10 anos. Com uma frota de 13,7 milhões de motocicletas, o Brasil está hoje no $4^{\circ}$ lugar do ranking de produtores mundiais de motocicletas, atrás apenas da China, Índia e Indonésia (ABRACICLO, 2010).

Além disso, este é um setor que têm introduzido no mercado, de forma sistemática, inovações e novos modelos. Isso torna este estudo de difusão de produtos de extrema importância para o setor, pois pretende contribuir para o enriquecimento tanto da literatura, especialmente colaborando com a aplicação de um modelo analítico, quanto da prática administrativa das empresas do setor.

A Tabela 1 apresenta os dados anuais de vendas de motocicletas no mercado brasileiro entre os anos de 1993 e 2010, obtidos do sitio da Associação Brasileira dos Fabricantes de Motocicletas, Ciclomotores, Motonetas, Bicicletas e Similares (ABRACICLO, 2010).

Nestes dados estão computadas as vendas agregadas de todos os modelos e fabricantes, todavia, a base de dados possui as vendas distribuídas por categoria (cilindradas), fabricante e modelo. Estes dados permitem estudar como algumas categoriais e marcas difundiram-se no mercado brasileiro, ou seja, permitem traçar um panorama do ciclo de vida desses modelos e categorias de motos utilizando-se o modelo de Frank Bass.

Tabela 1: Vendas anuais de motocicletas no Brasil
\begin{tabular}{|c|c|}
\hline Ano & Vendas de Motos \\
\hline 1993 & 67.997 \\
\hline 1994 & 120.962 \\
\hline 1995 & 200.592 \\
\hline 1996 & 275.668 \\
\hline 1997 & 404.635 \\
\hline 1998 & 460.122 \\
\hline 1999 & 441.536 \\
\hline 2000 & 574.149 \\
\hline 2001 & 692.096 \\
\hline 2002 & 792.429 \\
\hline 2003 & 848.377 \\
\hline 2004 & 911.717 \\
\hline 2005 & 1.024 .203 \\
\hline
\end{tabular}




\begin{tabular}{|l|l|}
\hline 2006 & 1.268 .041 \\
\hline 2007 & 1.600 .157 \\
\hline 2008 & 1.879 .695 \\
\hline 2009 & 1.579 .197 \\
\hline 2010 & 1.818 .049 \\
\hline
\end{tabular}

Fonte: (ABRACICLO, 2010)

\section{A}

Figura 3 ilustra a curva de crescimento deste mercado. Com uma taxa média de crescimento de $23 \%$ ao ano, esta curva revela a força do fenômeno de crescimento das vendas de motos no Brasil, dando uma dimensão da importância que estudos sobre o processo de difusão deste tipo de produto teriam para melhoria da capacidade competitiva das empresas.

Figura 3: Vendas anuais de motocicletas no Brasil

Venda de Motos (Mercado Interno)

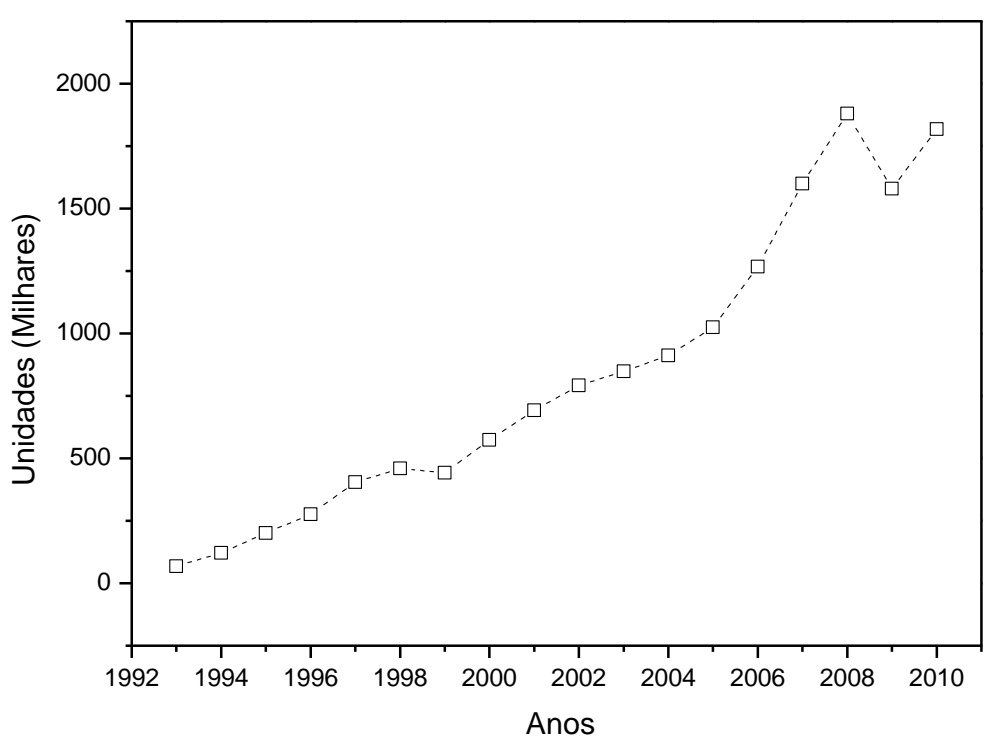

Fonte: Elaborado pelo autor

\section{Procedimentos metodológicos}

Esta pesquisa pode ser caracterizada como um estudo aplicado, já que visa gerar conhecimentos e modelos para aplicação em situações práticas. No que se refere ao método analítico, a pesquisa pode ser classificada como hipotético-dedutiva, pois o método consistirá na construção de conjecturas ou hipóteses representadas por modelos matemáticos. Os modelos podem ser então testados ou falseados por meio da comparação do comportamento destes modelos frente à realidade dos dados. As etapas do processo metodológico deste trabalho estão descritas abaixo:

a) Levantamento dos dados secundários: Os dados foram obtidos por meio do acesso aos bancos de dados da ABRACICLO (Associação Brasileira dos Fabricantes de Motocicletas, Ciclomotores, Motonetas, Bicicletas e Similares). Os bancos de dados contêm, entre outras 
informações, a evolução de vendas de diversas marcas e modelos de motocicletas no mercado Brasileiro;

b) Limpeza dos dados e construção do banco de dados de processamento: A atividade de limpeza de dados envolve a identificação e correção de eventuais falhas nas tabelas de dados que podem ter ocorrido durante o processo de digitação ou levantamento das informações. O objetivo é separar as tabelas que serão processadas;

c) Seleção dos dados de vendas das marcas e segmentos de produto para análise de difusão: Os resultados das etapas anteriores devem prover meios para que sejam selecionadas marcas e modelos de motocicletas candidatos a serem objetos da análise do ciclo de difusão. A ideia é analisar as marcas e modelos mais representativas do ponto de vista do volume de vendas e da extensão da comercialização no tempo;

d) Execução das análises de regressão dos dados de venda utilizando o modelo de Frank Bass: Nesta etapa serão realizados diversos ajustes de regressão utilizando o método de Mínimos Quadrados Não Lineares - MQNL (SEBER; WILD, 2003). Para o ajuste foi utilizado o software ORIGIN (ORIGINLAB, 2007). O objetivo será verificar quais processos de difusão (associados aos modelos escolhidos) podem ser explicados com o uso do modelo de Frank Bass;

e) Levantamento dos coeficientes de inovação, de imitação e de penetração de mercado para cada modelo de motocicleta escolhido: Os modelos ajustados serão analisados quanto à qualidade do ajuste e quanto aos valores dos coeficientes obtidos. Também serão feitos estudos comparativos dos coeficientes obtidos para cada modelo de motocicleta estudado.

\section{O modelo de Bass aplicado ao mercado de motos}

Para exemplificar como o modelo de Bass pode ser utilizado para estudar o ciclo de vida de difusão de modelos de motocicletas no Brasil, pode-se como exemplo a categoria de motos de 50cc. Estas motos, típicas para iniciantes ou para os que precisam da moto para pequenas atividades cotidianas, tornaram-se febre na década de 90, todavia, como usavam motores dois tempos, que usam gasolina misturada com óleo, esses motores produziam muita poluição e por esse motivo foram paulatinamente abandonados pela maioria dos fabricantes. $\mathrm{O}$ fato desta categoria $(50 \mathrm{cc})$ ter a fase de crescimento na década de 90 , entrando em declínio nos anos 2000 , permite que se trabalhe com um ciclo completo, o que torna a análise mais abrangente para esta categoria.

$\mathrm{Na}$ Figura 4 estão representadas as vendas anuais consolidadas (todos os fabricantes e modelos) de motocicletas de 50cc no Brasil de 1993 até 2006, que é o último ano de registro nos dados da ABRACICLO.

É possivel notar que a curva apresenta o perfil típico de uma curva completa de difusão. Podem-se obter os coeficientes do modelo de Bass desta curva realizando um ajuste da Equação (5) 
por meio do método MNQL. Na Figura 4 a curva ajustada $\left(R^{2}=0.87\right)$ representa o modelo de Bass ajustado para os dados consolidados das motos de 50cc. Os coeficientes $p, q$ e $m$ obtidos não podem ser interpretados isoladamente. Eles devem ser comparados com outros coeficientes para que se tenha uma ideia objetiva a respeito de seus valores.

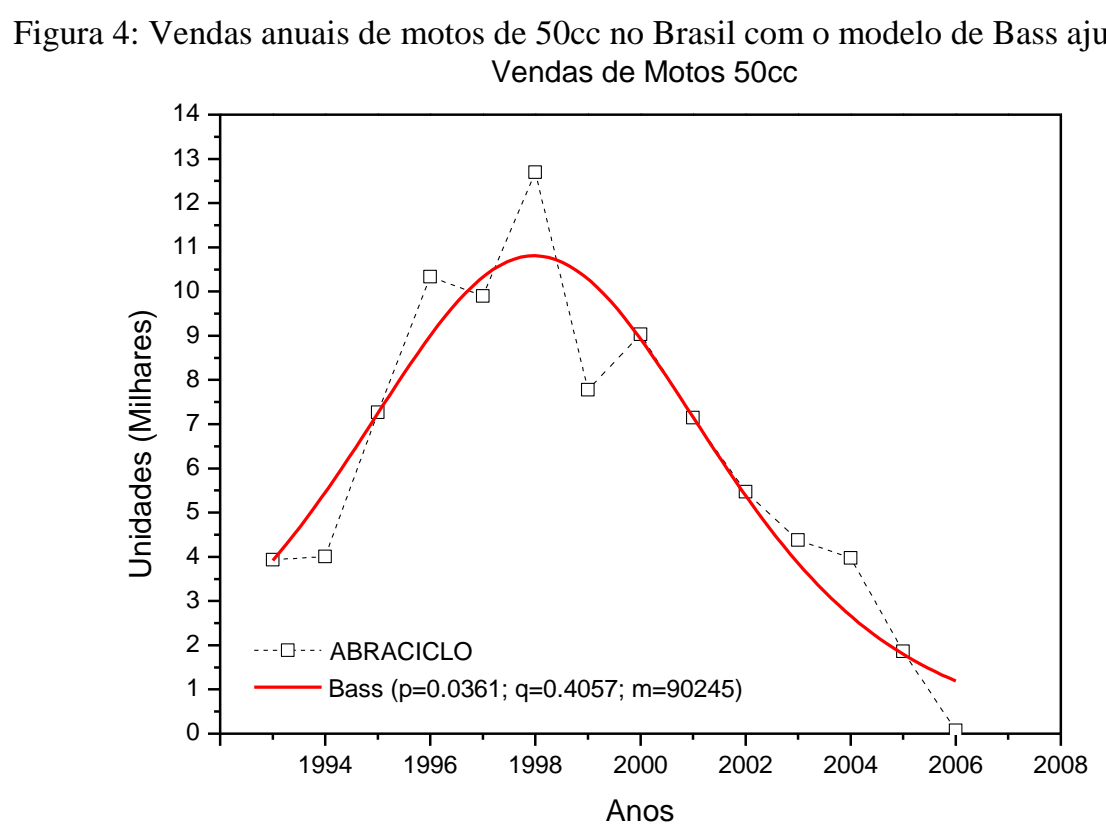

Fonte: Elaborado pelo autor

É possível analisar os modelos de um determinado fabricante nesta categoria e comparar os valores dos modelos de outros fabricantes. Tome como exemplo a fabricante Yamaha que, segundo os dados da ABRACICLO, de 1993 a 2006 foi responsável por 53\% das vendas de motocicletas de 50cc. As demais fabricantes, Brandy, Caloi, Sundow, Cofave e Kasinski foram responsáveis juntas pelos restantes das vendas $(47 \%)$.

$\mathrm{Na}$ Figura 5 são exibidas as curvas de vendas anuais dos três modelos de motos comercializados pela Yamaha nos período de 1993 a 2006: BW’S 50, JOG 50 e JOG TEEN 50. As três curvas apresentam sinais de ciclos característicos de crescimento e declínio. Pode-se perceber também que devido ao fato da JOG TEEN 50 ser uma extensão da linha JOG, a curva da JOG TEEN 50 é aparentemente uma extensão do ciclo da JOG 50, ou seja, a soma das duas curvas forma uma única curva de difusão para a família JOG. Isto se deve ao fato de que entre os produtos houve apenas uma transição, com mudanças de apenas pequenas características.

Cada uma das três curvas da Figura 5 pode ser ajustada com um modelo de Bass para que os coeficientes de cada modelo possam ser comparados. Os resultados dos ajustes estão sintetizados na Tabela 2.

Nesta tabela percebe-se que o modelo JOG 50 foi o que apresentou o maior coeficiente de inovação (p). Isto se deve provavelmente ao fato deste modelo ter sido o primeiro a ser lançado e, 
por não contar com uma base pregressa de usuários, teve grande esforço de comunicação, o que fez com que as pessoas fossem levadas a adotar o produto motivadas basicamente pelo apelo inovador e pelo esforço de comunicação. Já a segunda geração desta motocicleta, JOG TEEN 50, tem uma curva de difusão com um menor coeficiente de inovação e um maior coeficiente de imitação. Isto leva a crer que este modelo tem o processo de adoção muito mais associado ao contágio social de mercado, ou seja, por uma pressão gerada pelo testemunho de usuários pregressos da JOG 50.

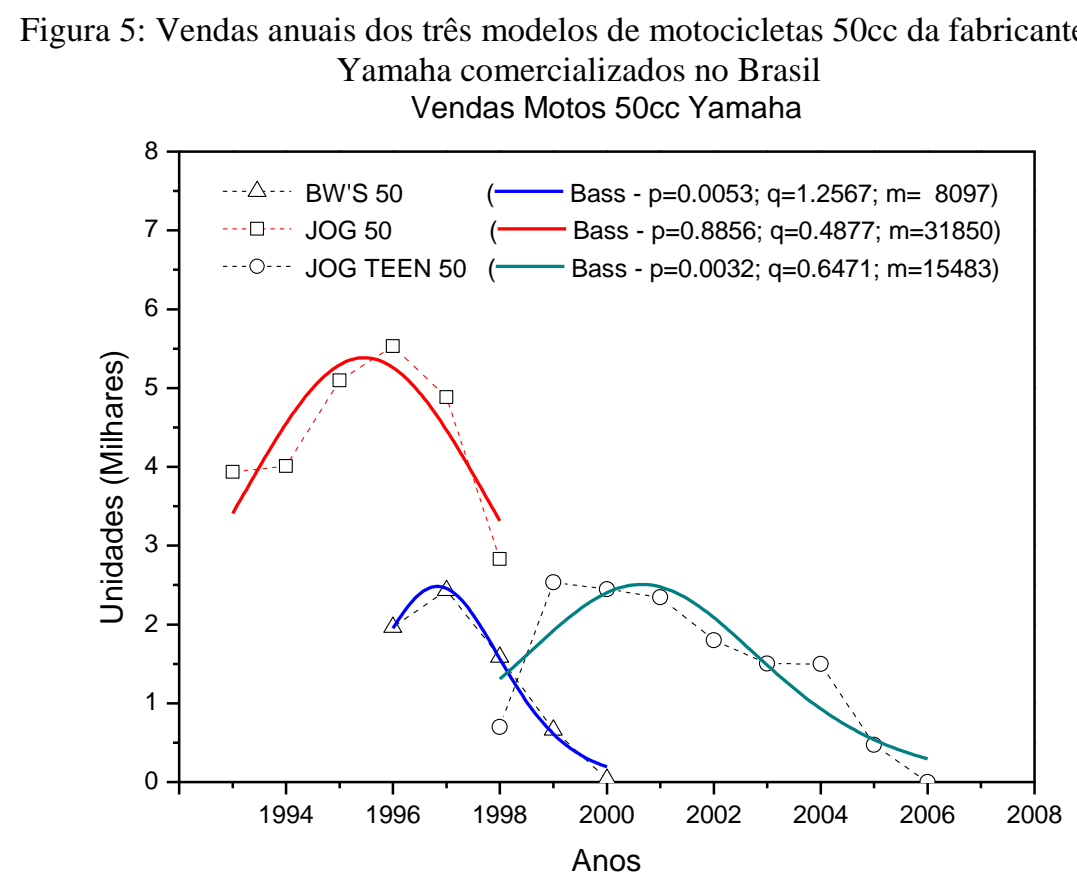

Fonte: Elaborado pelo autor

Tabela 2: Coeficientes do modelo de Bass obtidos a partir das curvas apresentadas na Figura 5 para cada um dos modelos de 50cc da Yamaha

\begin{tabular}{|l|c|c|c|c|}
\hline \multicolumn{1}{|c|}{ Modelo } & $\boldsymbol{p}$ & $\boldsymbol{q}$ & $\boldsymbol{m}$ & Período \\
\hline JOG 50 & 0,0886 & 0,4877 & 31851 & $1993-1998$ \\
\hline JOG TEEN 50 & 0,0032 & 0,6471 & 15484 & $1998-2006$ \\
\hline BW's 50 & 0,0053 & 1,2567 & 8097 & $1996-2000$ \\
\hline
\end{tabular}

Fonte: Elaborado pelo autor

O modelo BW's 50, assim como o modelo JOG TEEN 50, têm um coeficiente de inovação menor que o modelo JOG TEEN. Porém, o coeficiente de imitação é maior que os outros dois modelos, caracterizando uma pressão de adoção social maior do que os demais.

Pode-se também analisar as vendas conjuntas dos demais fabricantes utilizando-se o modelo de Bass. Na Figura 6 estão representadas as vendas anuais consolidadas dos modelos de motocicletas 50cc dos fabricantes, Brandy, Caloi, Sundow, Cofave e Kasinski Yamaha comercializados no Brasil juntamente com o modelo de Bass ajustado. 
Figura 6: Vendas anuais consolidadas dos modelos de motocicletas 50cc dos fabricantes: Brandy,

Caloi, Sundow, Cofave e Kasinski Yamaha com o modelo de Bass ajustado Vendas Motos 50cc (Brandy/Caloi/Sundow/Cofave/Kasinski)

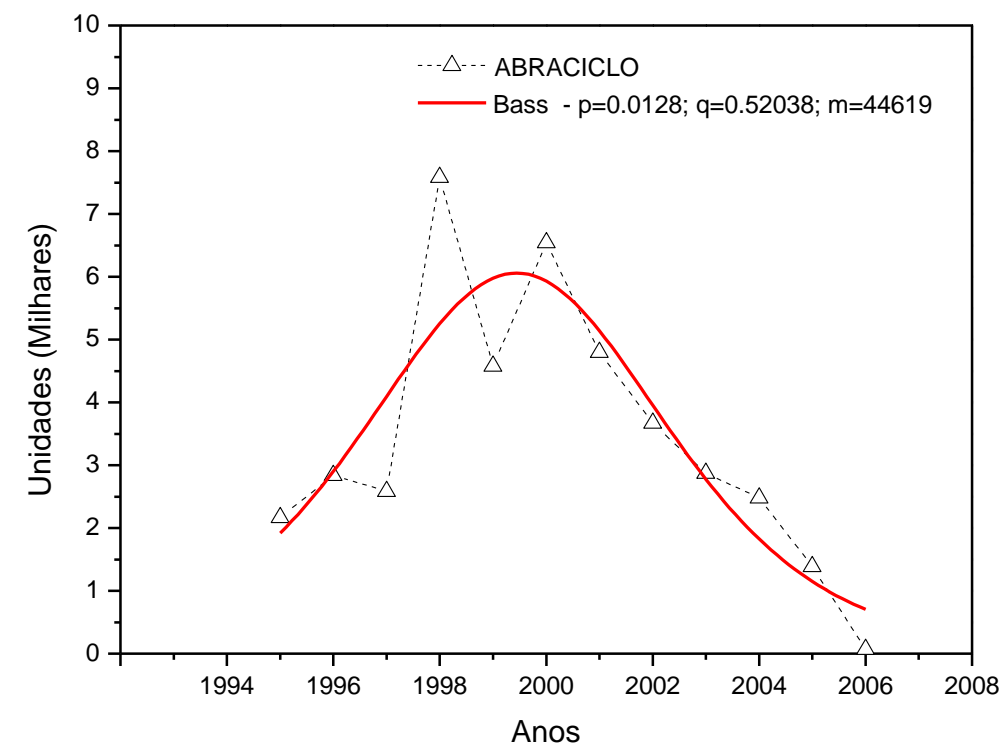

Fonte: Elaborado pelo autor

Na Figura 7 estão representadas as vendas anuais de motocicletas $600 \mathrm{cc}$ no Brasil. Percebese que esta curva é mais complexa do que a que foi apresentada na Figura 4, pois apresenta um conjunto maior de máximos e de mínimos. Isto acontece porque a curva é composta de múltiplos ciclos de difusão, que têm de ser modelados de forma independente para que se possam representar as vendas agregadas da categoria. Entre os anos de 1998 e 2010 apenas dois fabricantes de motos são listados na ABRACICLO como fabricantes nesta categoria: Honda e Yamaha.

Figura 7: Vendas anuais de motocicletas 600cc no Brasil (Fonte: ABRACICLO) Vendas de Motos $600 \mathrm{cc}$

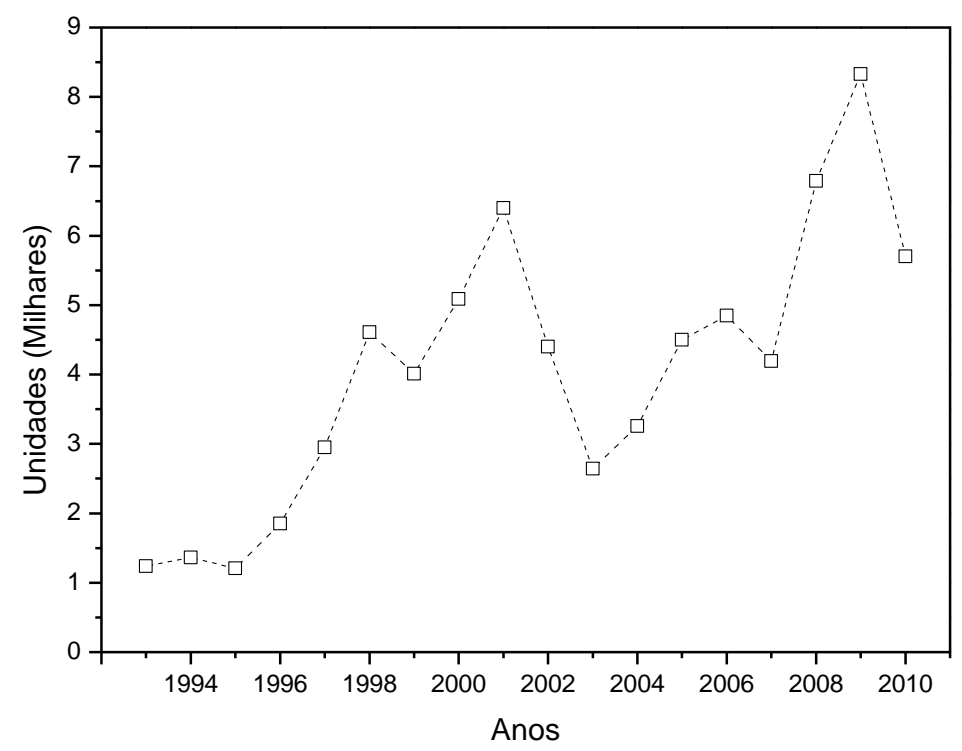

Fonte: Elaborado pelo autor 
$\mathrm{Na}$ Figura 8 estão representadas as vendas anuais de dois modelos de 600cc comercializados pela Honda: Hornet 600F (nas versões padrão e ABS) e VT 600 (todas as versões). Pode-se perceber que há um padrão de difusão em formato de "ciclo de vida" em cada uma das curvas.

Figura 8: Vendas anuais dos dois modelos de motocicletas $600 \mathrm{cc}$ da fabricante Honda comercializados no Brasil Vendas Motos 600cc Honda

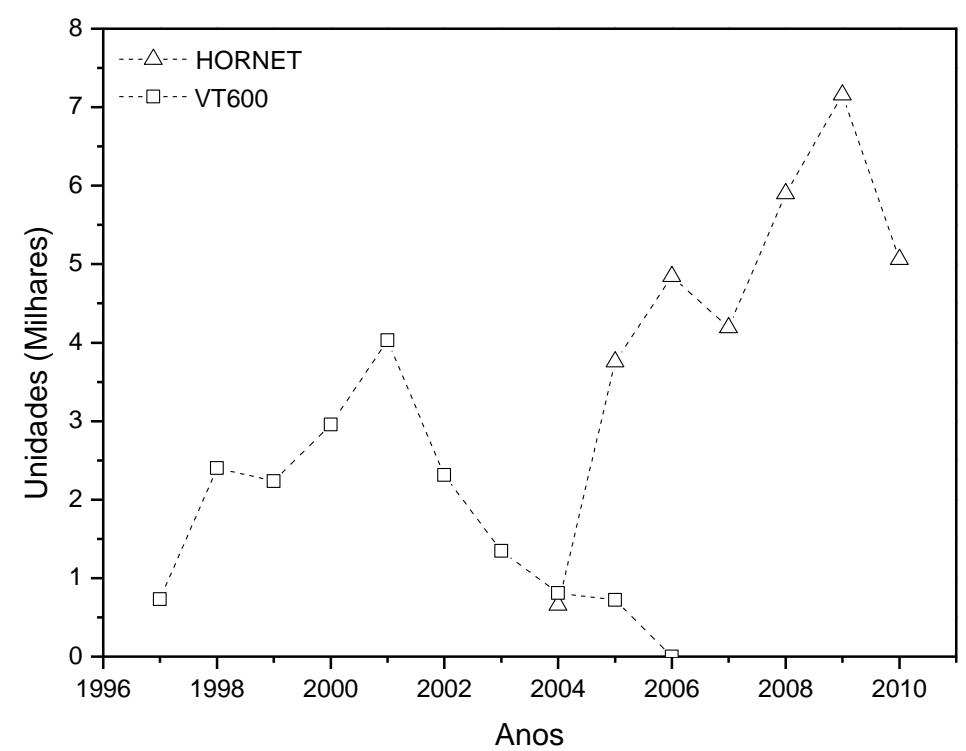

Fonte: Elaborado pelo autor

A

Figura 9 mostra a equação de Bass ajustada para estes dois modelos de moto.

Figura 9: Vendas anuais dos dois modelos de motocicletas $600 \mathrm{cc}$ da fabricante Honda comercializados no Brasil e o modelo de Bass ajustado Vendas Motos 600cc Honda

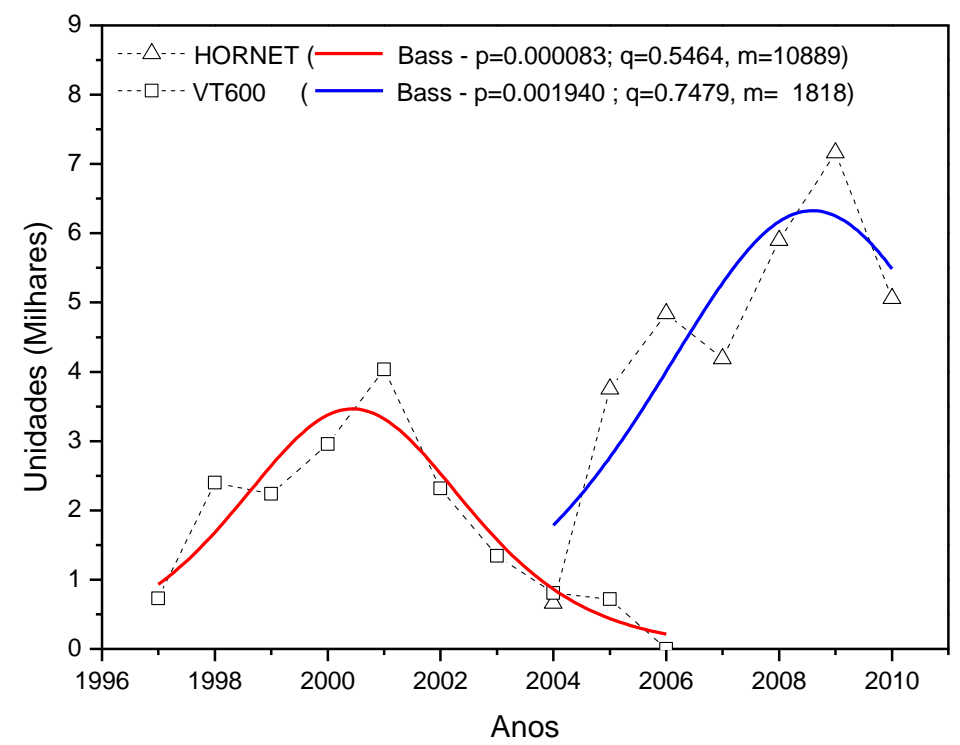

Fonte: Elaborado pelo autor 
Em particular, nota-se na

Figura 9 que o modelo VT600 possui um coeficiente de inovação significativamente maior que o coeficiente de inovação do modelo Hornet. O ciclo de vida do modelo VT600 apresenta todas as fases, chegando até o declínio. Nota-se também que o modelo Hornet, que foi introduzido em 2004, substitui o modelo VT600. Isto significa que boa parte do estímulo de venda do modelo Hornet pode ser devido a impressões e influências de usuários pregressos do modelo VT600, o que explica em parte o fato do modelo Hornet ter o processo de difusão muito mais influenciado por aspectos relacionados à influência social.

A Yamaha é o outro fabricante de moto na categoria de $600 \mathrm{cc}$ registrada nas bases de dados da ABRACICLO no período de 1993 a 2010. A Figura 10 apresenta as curvas de vendas dos modelos XT e XJ 600 e FAZER. A FAZER, introduzida em 2008, possui registro de apenas 3 anos de vendas. Dessa forma, não é possível fazer o ajuste da equação de Bass, pois Srinivasan e Mason (1986) mostraram que as estimativas do modelo de Bass ficam comprometidas se menos de 4 períodos anuais estão disponíveis para o ajuste.

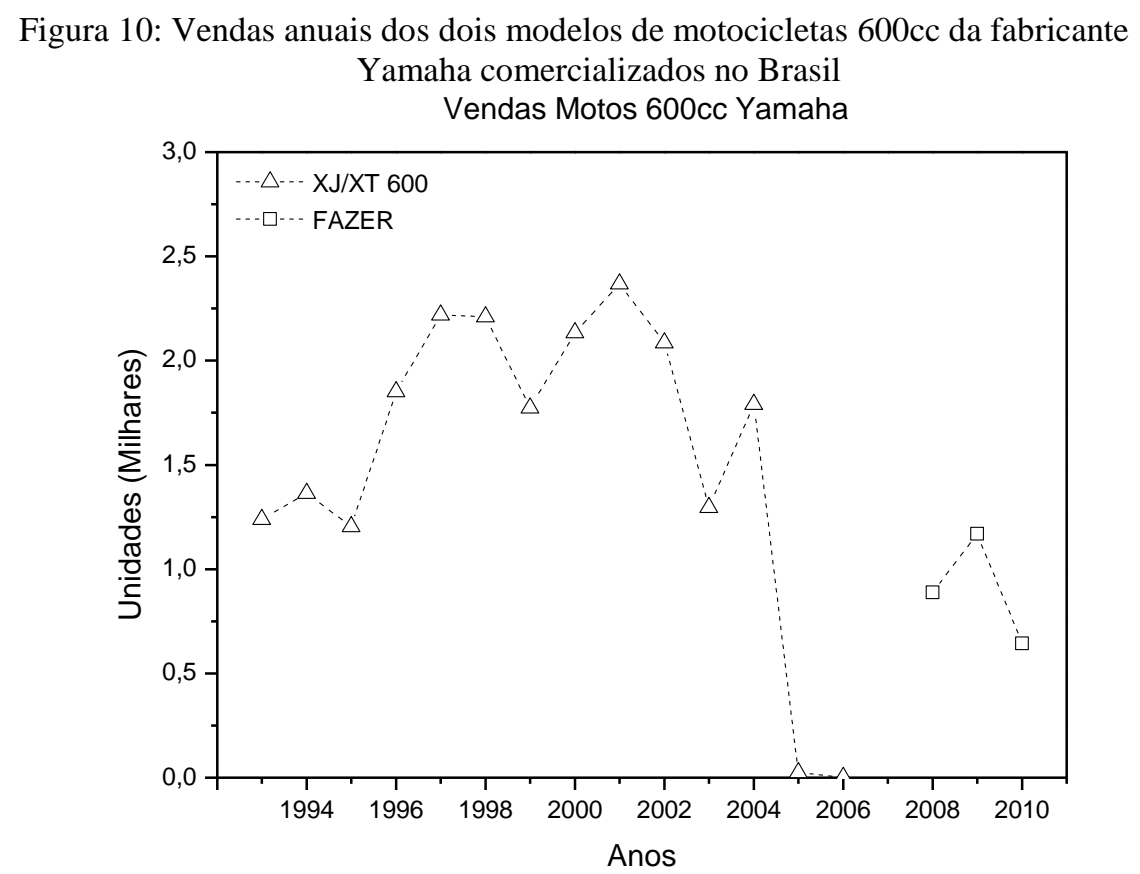

Fonte: Elaborado pelo autor

Todavia, para os modelos XT/XJ 600 é possível fazer o ajuste da curva de Bass. A Figura 11 mostra o resultado deste ajuste e os coeficientes obtidos. Para obter-se o modelo que ajusta a curva total de vendas apresentado na Figura 7 deve-se somar as curvas ajustadas para os modelos da Honda (Hornet e VT 600) e Yamaha (XT/XJ 600). 
Figura 11: Vendas anuais dos dois modelos de motocicletas $600 \mathrm{cc}$ da fabricante Honda comercializados no Brasil e o modelo de Bass ajustado Vendas Motos 600cc Yamaha

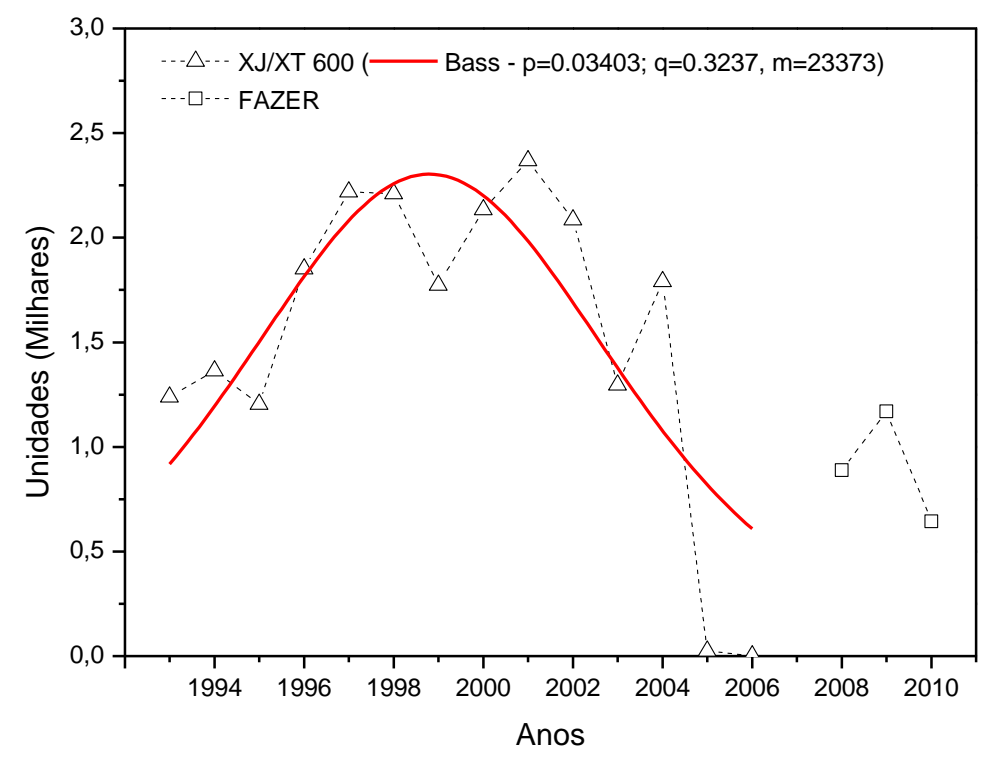

Fonte: Elaborado pelo autor

Na Figura 12 é apresentada a soma das curvas ajustadas para os modelos da Honda (Hornet e VT 600) e Yamaha (XT/XJ 600).

Figura 12: Vendas anuais dos dois modelos de motocicletas $600 \mathrm{cc}$ da fabricante Yamaha comercializados no Brasil Vendas de Motos $600 \mathrm{cc}$

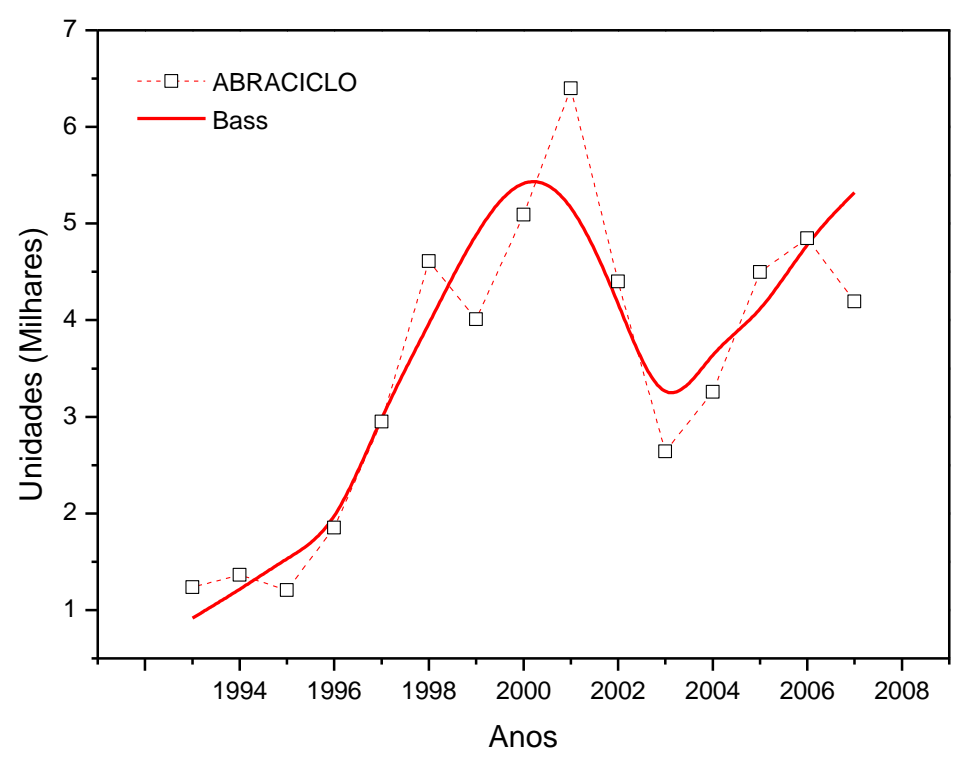

Fonte: Elaborado pelo autor

Observa-se nesta figura que as curvas ajustadas para cada modelo, quando somadas, geram uma curva que ajusta os dados consolidados da categoria $\left(R^{2}=0.87\right)$. Desta forma, percebe-se que as curvas de difusão consolidadas podem ser obtidas por meio do ajuste das curvas de cada categoria e produto. Os fabricantes podem então ter uma melhor ideia da evolução futura de suas 
vendas fazendo o acompanhamento e a previsão da difusão de cada produto por meio do modelo de Bass.

\section{Discussão dos resultados}

A Tabela 3 apresenta um resumo dos coeficientes obtidos nos ajustes da equação de Bass que foram feitos para os modelos de motos estudados neste trabalho. Esta tabela pode ser representada na forma de um gráfico onde cada ponto representa um modelo de moto no espaço de fase dos coeficientes de inovação e imitação. Este gráfico (Figura 13) permite uma análise visual de como os parâmetros dos modelos de um mesmo segmento se agrupam.

Tabela 3: Resumo dos coeficientes obtidos nos ajustes da equação de Bass feitos para os modelos de motos estudados neste trabalho

\begin{tabular}{|l|c|c|c|c|}
\hline \multicolumn{1}{|c|}{ Modelo } & $\boldsymbol{p}$ & $\boldsymbol{q}$ & $\boldsymbol{m}$ & Período \\
\hline Yamaha JOG 50 & 0,0886 & 0,4877 & 31851 & $1993-1998$ \\
\hline Yamaha JOG TEEN 50 & 0,0032 & 0,6471 & 15484 & $1998-2006$ \\
\hline Yamaha BW's 50 & 0,0053 & 1,2567 & 8097 & $1996-2000$ \\
\hline Yamaha XT/XJ 600 & 0,03403 & 0,3237 & 23373 & $1993-2006$ \\
\hline Honda Hornet 600 & 0.000083 & 0.5464 & 10889 & $2004-2010$ \\
\hline Honda VT 600 & 0,00194 & 0,7479 & 1818 & $1997-2006$ \\
\hline
\end{tabular}

Fonte: Elaborado pelo autor

Figura 13: Gráfico $\quad p \times q$ onde os pontos representam cada um dos

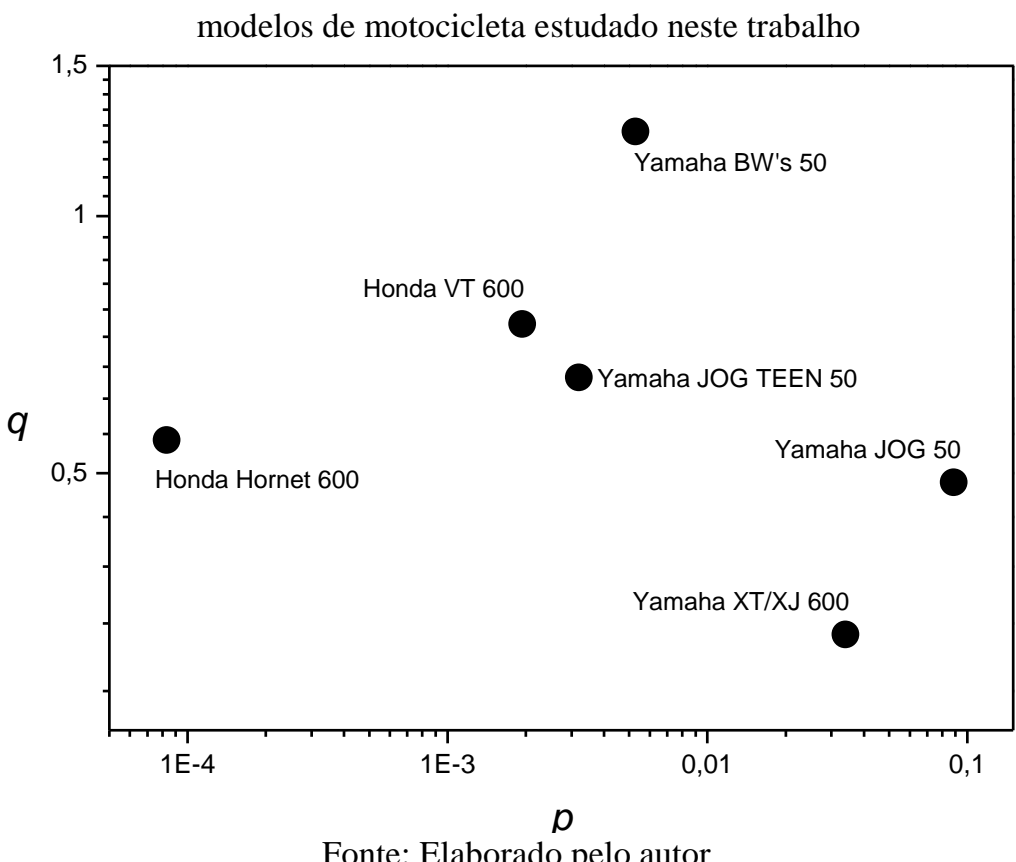

Fonte: Elaborado pelo autor

O estudo dos coeficientes é de extrema importância na análise dos ajustes. No modelo de Bass, o coeficiente $p$ captura a influência sobre os potenciais adotantes que podem ser considerados independentes em relação à opinião dos adotantes já existentes em um mercado. Isto é, a influência 
capturada pelo coeficiente de inovação não é derivada do processo tradicional de comunicação interpessoal (boca a boca) (HORSKY; SIMON, 1983).

O valor médio do coeficiente de inovação para novos produtos varia de forma geral entre $p=0.00007$ e $p=0.03$ (SULTAN; FARLEY et al., 1990; TALUKDAR; SUDHIR et al., 2002; VAN DEN BULTE; STREMERSCH, 2004), o que se enquadra nos valores obtidos neste trabalho. A influência capturada por este coeficiente deriva basicamente de dois aspectos, tomados em relação a um determinado mercado: (1) o acesso que o consumidor tem a informações sobre o produto ou a questões relacionadas a ele (obtidas sem o uso de boca a boca) e; (2) a habilidade que os consumidores do país têm para processar informação de outros canais de comunicação, que não o boca a boca (TALUKDAR; SUDHIR et al., 2002).

No caso do acesso do consumidor a informações relacionadas ao produto, é importante lembrar que os consumidores podem interagir com outras pessoas por meio de telefone, e-mail etc. Ou usar TV, jornais e outros canais de mídia de massa para obter acesso à informação que esteja relacionada a um determinado produto. $\mathrm{O}$ acesso do consumidor a informações relacionadas ao produto tem relação direta com o tempo decorrido desde que um produto foi lançado no mercado. Quanto maior for este tempo, mais os consumidores estarão propensos a tentar obter informações sobre ele (DEKIMPE; PARKER et al., 2000), fazendo com que a penetração de mercado do produto aumente ao longo dos anos, desde sua introdução.

Além disso, a habilidade dos consumidores em processar informações de fontes que não o boca a boca também é um fator importante a ser considerado na composição do coeficiente de inovação. Consumidores com maior nível educacional são mais propensos a procurar informação, de forma independente, sobre os benefícios de um produto (HIRSCHMAN, 1980). Estes consumidores são mais inclinados a utilizar outras formas e canais de comunicação que não o tradicional boca a boca para fazer julgamentos sobre os novos produtos. É o que ocorre normalmente para produtos de maior valor agregado.

Enquanto o coeficiente de inovação $p$ na Tabela 3 captura uma tendência autônoma (intrínseca) do indivíduo em adotar um dado modelo de moto, o coeficiente de imitação $q$ mede a influência que um conjunto existente de consumidores exerce sobre as decisões de compra de outros potenciais consumidores, que ainda não adotaram o modelo.

$\mathrm{O}$ valor médio do coeficiente de imitação para novos produtos varia em geral entre $q=0.38 \mathrm{e}$ $q=0.53$ (SULTAN; FARLEY et al., 1990; TALUKDAR; SUDHIR et al., 2002; VAN DEN BULTE; STREMERSCH, 2004). Pode-se notar, observando a Tabela 3, que praticamente todos os modelos de motos estudados (excetuando-se o modelo Yamaha XT/XJ 600) ficaram fora e acima desta faixa. Isto demonstra o papel de importância que a comunicação interpessoal tem no mercado de motos, no sentido de influenciar e criar novos consumidores. 
O coeficiente de imitação $q$ captura a efetividade da comunicação interpessoal como elemento propulsor da difusão. A literatura mostra que este coeficiente está basicamente relacionado com: (1) o nível de homogeneidade cultural da população e; (2) com a capacidade de persuasão dos consumidores que já adotaram o produto (TALUKDAR; SUDHIR et al., 2002).

O coeficiente de imitação $q$ é esperado aumentar em um mercado onde é maior a capacidade de persuasão das recomendações boca a boca dos adotantes existentes. Essa capacidade de persuasão das recomendações aumenta com uma maior familiaridade e satisfação dos atuais adotantes com o novo produto (TAKADA; JAIN, 1991). Talukdar, Sudhir e Ainslie (2002) representam o nível de satisfação e familiaridade dos adotantes de um mercado em termos do número de anos que a introdução do produto tem de atraso dos mercados lideres, por exemplo, de outros países. A hipótese é a de que um produto que tenha estado no mercado por vários anos, tem mais chances de ter tido seus erros e falhas corrigidos, e de ter desenvolvido uma longa curva de aprendizado com os fabricantes. Isto é o que acontece com modelos subsequentes de motos, por exemplo, JOG e JOG TEEN.

Muitos outros testes podem ser feitos a partir de novas simulações e ajustes do modelo de Frank Bass aplicado aos dados do setor estudado. Todavia, como é normal neste tipo de desenvolvimento, há algumas limitações que têm de ser consideradas, principalmente para aqueles que venham a trabalhar na extensão dos conceitos e ideias aqui apresentados. Uma das maiores restrições está na perfeita determinação dos parâmetros. Neste trabalho, foi deixado que um ajuste numérico em relação aos dados disponíveis fornecesse o valor dos parâmetros, porém, os valores obtidos devem ser mais bem investigados sob outras perspectivas, que incluam ferramentas de pesquisa mercadológica e econométricas clássicas. Isso dará mais robustez aos resultados obtidos ou então permitirá o surgimento uma crítica compreensiva dos fenômenos aqui descritos.

Também devem ser considerados outros aspectos econômicos que não foram considerados para construção dos cenários, por exemplo, aqueles referentes às eventuais políticas regulatórias do país. Tais aspectos têm impacto sobre o processo de difusão de motocicletas na medida em que perturbam a oferta. Essa perturbação pode ocorrer por meio da liberação de ofertas complementares (importação) ou por meio do bloqueio dessas ofertas (barreiras). Isso pode elevar o benefício de uso de um produto, por exemplo, devido à escassez e a exclusividade de um número limitado de fabricantes. Todos estes fatores devem ser considerados em pesquisas futuras sobre este tema.

Por fim, todas estas observações, e muitas outras tratadas na literatura sobre o tema, permitem aos gestores a definição de formas mais seguras e objetivas para lidar com suas expectativas relativas a projetos que envolvam a difusão de novos produtos. A objetividade nas análises deve ser buscada. Stremersh e Tellis (2004) comentam, por exemplo, que os gestores tendem a subestimar o tempo que um produto leva para iniciar a fase de crescimento em um 
mercado, chamado na literatura de takeoff. E tendem a superestimar o tempo para atingir a fase de maturidade e a saturação das vendas. Análises mais estruturadas do processo de difusão de produtos poderiam evitar, ou pelo menos ajudar a minimizar, estes e outros tipos de problemas na formulação de estratégias de expansão.

\section{Referências}

ABRACICLO. Estatística de produção e vendas de motocicletas. São Paulo, 2010. Disponível em: < www.abraciclo.com.br >.

ANFAVEA. Estatística de produção por tipo, empresa e modelo. São Paulo, 2010. Disponível em: < www.anfavea.com.br $>$.

BASS, F. M. A New Product Growth Model for Consumer Durables. Management Science, v. 15, n. 5, p. 215-227, 1969.

\section{crost}

CHANDRASEKARAN, D.; TELLIS, G. J. Diffusion of new products: a critical review of models, drivers, and findings. Review of Marketing Research, v. 3, p. 39-80, 2006.

DEKIMPE, M.; PARKER, P.; SARVARY, M. Multimarket and global diffusion. In: MAHAJAN, V.; MULLER, E. et $a l$. (Ed.). New product diffusion models. Boston: Kluwer Academic, 2000.

FIGUEIREDO, J. C. B. Difusão de produtos através de países. In: AMATUCCI, M. (Ed.). Internacionalização de Empresas: teoria, problemas e casos. 1 ed. São Paulo: ATLAS, 2009a. cap. 4, p.183-214.

Estudo da difusão da tecnologia móvel celular no Brasil: uma abordagem com o uso de Dinâmica de Sistemas. Produção, v. 19, n. 1, p. 230-245, 2009b.

FOURT, L.; WOODLOCK, J. Early prediction of market success for new grocery products. The Journal of Marketing, v. 25, n. 2, p. 31-38, 1960.

HEELER, R.; HUSTAD, T. Problems in predicting new product growth for consumer durables. Management Science, v. 26, n. 10, p. 1007-1020, 1980.

\section{cross}

HIRSCHMAN, E. C. Innovativeness, novelty seeking and consumer creativity. J. Consumer Res., v. 7, p. 283-295, 1980.

\section{crosst}

HORSKY, D.; SIMON, L. S. Advertising and the diffusion of new products. Marketing Science, v. 2, n. 1, p. 1-17, 1983. 
KALISH, S. A new product adoption model with pricing, advertising and uncertainty. Management Science, v. 31 , p. $1569-1585,1985$.

\section{crost}

KALISH, S.; LILIEN, G. A market entry timing model for new technologies. Management Science, v. 32, n. 2, p. 194205, 1986.

\section{cross ref}

MAHAJAN, V.; MULLER, E.; BASS, F. New product diffusion models in marketing: A review and directions for research. The Journal of Marketing, v. 54, n. 1, p. 1-26, 1990.

\section{crost}

MAHAJAN, V.; MULLER, E.; BASS, F. M. New product diffusion models in marketing: a review and directions for research. Journal of Marketing, v. 54, p. 1-26, 1990.

crost

MANSFIELD, E. Technical change and the rate of imitation. Econometrica: Journal of the Econometric Society, v. 29 , n. 4, p. 741-766, 1961.

\section{crost ref}

ORIGINLAB. Origin: getting started manual version 8. 2007. Disponível em: <www.originlab.com>. Acesso em: 22/03/2012.

PERES, R.; MULLER, E.; MAHAJAN, V. Innovation diffusion and new product growth models: a critical review and research directions. International Journal of Research in Marketing, vol. April, 2010. Disponível em: < http://linkinghub.elsevier.com/retrieve/pii/S0167811610000236 >.

ROGERS, E. Diffusion of innovations. New York: Free Press, 1995.

SATOH, D. A discrete bass model and its parameter estimation. Journal of the Operations Research Society of Japan-Keiei Kagaku, v. 44, n. 1, p. 1-18, 2001.

SEBER, G. A. F.; WILD, C. J. Nonlinear regression. LibreDigital, 2003.

SRINIVASAN, V.; MASON, C. Nonlinear least squares estimation of new product diffusion models. Marketing Science, v. 5, n. 2, p. 169-178, 1986.

\section{crost}

STONEMAN, P. The economics of technological diffusion. Cambridge: Blackwell, 2002.

STREMERSCH, S.; TELLIS, G. J. Understanding and managing international growth of new products. Intern. J. of Research in Marketing v. 21, p. 421-438, 2004. 
SULTAN, F.; FARLEY, J. U.; LEHMANN, D. R. A meta-analysis of diffusion models. Journal of Marketing Research, v. 27, p. 70-77, 1990.

\section{erasef}

TAKADA, H.; JAIN, D. Cross-national analysis of diffusion of consumer durable goods in pacific rim countries. J. Marketing, v. 55, p. 48-54, 1991.

\section{erpst}

TALUKDAR, D.; SUDHIR, K.; AINSLIE, A. Investigating new product diffusion across products and countries. Marketing Science, v. 21, n. 1, p. 97-114, 2002.

\section{crost}

VAN DEN BULTE, C.; STREMERSCH, S. Social contagion and income heterogeneity in new product diffusion: a meta-analytic test. Marketing Science, v. 23, n. 4, p. 530-544, 2004

\section{crost}

VASCONCELlOS, E. O custo social da motocicleta no Brasil. Revista dos Transportes Públicos-ANTP, v. 30, 2008.

WRIGHT, M.; CHARIETT, D. New product diffusion models in marketing: an assessment of two approaches. Marketing Bulletin, v. 6, p. 32-41, 1995.

\section{Dados do autor:}

\section{Nome completo: Júlio César Bastos de Figueiredo}

Filiação institucional: Escola Superior de Propaganda e Marketing

Departamento: Programa de Mestrado em Gestão Internacional

Função ou cargo ocupado: Professor Titular

Endereço completo para correspondência: Rua Padre Raposo, 1300, Apto 202, São Paulo, SP,

Brasil - 03118-001

Telefones para contato: (11) 9162-6770; (11) 5081-8176

e-mail: jfigueiredo@espm.br

\section{Enviado em: 03/06/2011}

Aprovado em: 08/03/2012 